\title{
Análisis de la influencia de la temperatura en la demanda residencial de energía eléctrica en Andalucía
}

Ester GUTIÉRREZ MOYA*

María Teresa ARÉVALO QUIJADA**

\section{RESUMEN}

Como consecuencia de las restricciones del suministro eléctrico, en los últimos años, la debilidad del sistema eléctrico español se ha convertido en objeto de discusión por diversos agentes económicos. Esta situación tiene lugar en determinadas épocas del año en las que se incrementa considerablemente el consumo de energía eléctrica debido, principalmente, a las variaciones imprevistas y a los niveles extremos que registran las temperaturas. Andalucía ha sido una de las comunidades afectadas por estas circunstancias, persistiendo el riesgo de seguir padeciendo sus consecuencias. En este trabajo se analiza el impacto que ejerce la temperatura ambiente sobre en el consumo residencial mensual de energía eléctrica a través de modelos basados en series cronológicas que expliquen su influencia y proporcionen estimaciones precisas de los niveles de demanda.

\section{PALABRAS CLAVE:}

Demanda de electricidad, grados mensuales, modelos de previsión.

* Departamento de Organización Industrial y Gestión de Empresas. Escuela Superior de Ingenieros. Universidad de Sevilla

** Departamento de Economía Aplicada III . Facultad de Ciencias Económicas y Empresariales. Universidad de Sevilla 


\section{ABSTRACT}

Over the last few years, the weakness of the Spanish electrical system has been object of discussion by diverse economic agents as a result of the restrictions in the electrical supply. This situation occurs at certain times of the year in which the consumption of electrical power is increased considerably mainly due to unexpected variations and extreme levels of the temperatures. Andalusia has been one of the communities affected by these circumstances and the risk of being affected still exists. This paper analyses the impact of temperature on the monthly residential consumption of electrical power through models based on chronological series that study their influence and provide accurate estimations of demand levels.

\section{KEYWORDS:}

Electricity demand, degree monthly, forecasting models.

UNESCO Code: 5302.02, 5312.05, 1209.15 JEL Code: Q41

\section{INTRODUCCIÓN}

La energía es uno de los pilares básicos del desarrollo social y económico de los países, siendo necesarias acciones orientadas a asegurar el abastecimiento, el respeto del entorno, el incremento de la competitividad y la diversificación de las fuentes de suministro. Actualmente, el sistema eléctrico español presenta uno de los niveles de dependencia energética más elevados de Europa, convirtiéndole en un mercado sensible frente a variaciones de los factores de carácter económico, social y meteorológico, entre otros.

El suministro de electricidad puede resultar deficitario ante situaciones meteorológicas desfavorables e imprevistas, siendo considerada la temperatura ambiental un componente básico y de especial relevancia en el análisis de la demanda de la energía eléctrica. Además, en los últimos años la temperatura, como factor de riesgo, ha adquirido mayor importancia en un escenario global de notable cambio climático. 
La influencia de las condiciones meteorológicas en la demanda de electricidad ha sido estudiada por diversos autores, pudiendo citarse entre los primeros que se ocuparon de este tema a Dryar, H.A. (1944), Williams y Leslie (1953), Davies, M. (1959), Quayle, R.G. y Diaz, H.F. (1980). En la última década también han sido varios autores los que han trabajado y propuesto diversas aproximaciones relacionadas con la temperatura, tales como las basadas en los splines, Harvey, A.C. y Koopman, S.J. (1993), modelos de regresión múltiple, Engle, R. et al. (1997) y las fundamentadas en redes neuronales artificiales, Hipper, H.S. et al. (2001), no llegándose a un consenso acerca del modelo más adecuado. La demanda de energía eléctrica en España ha sido tratada por diversos autores, entre los que se podrían citar, a Sanz, R. (1979), Gallástegui, I. (1986), Canceló, J.R y Espasa, A. (1991), entre otros; sin embargo, el número de estudios que desarrollan su análisis a partir de la temperatura es reducido, destacando en los últimos años el trabajo de Canceló, J.R. y Espasa, A. (1995), donde aplican un procedimiento basado en funciones de transferencia.

En el estudio de la demanda de energía eléctrica conviene segmentar los kilovatios por hora en los que se observa un comportamiento similar, no existiendo un criterio uniforme aceptado. Sin embargo, una clasificación tradicionalmente empleada es la que divide el consumo según la actividad o uso al que se destine. La demanda de energía eléctrica en Andalucía se debe principalmente a tres tipos de clientes; éstos son los procedentes del sector industrial, comercial y residencial. La demanda de electricidad derivada de la actividad agrícola, construcción y alumbrado público tiene un carácter residual frente a la demanda total, representando aproximadamente un 3,07\%, según los datos del año 2002.

En los últimos años, el consumo de energía eléctrica en Andalucía se ha caracterizado por un crecimiento continuado en los tres principales sectores demandantes, a excepción del año 2002, en el que desciende el consumo industrial un $1,70 \%$, tal como se puede observar en la cuadro 5 , donde se muestran los datos de la demanda de energía eléctrica en Andalucía segmentada durante el periodo 19952002. El sector residencial es el que ha sufrido un mayor crecimiento alcanzando una tasa media anual del 7,50\%, seguido del sector comercial, con una tasa del 5,93\%, y el sector industrial, con un $3,34 \%$. 
La demanda de energía eléctrica en Andalucía posee una estructura equilibrada donde la cuota sectorial oscila alrededor de la tercera parte de la demanda total. La contribución del sector industrial a la demanda final ha sufrido una disminución del $5,16 \%$ desde el año 1995 en favor, principalmente, del sector residencial, con un crecimiento del $4,23 \%$, ya que la cuota de participación del sector comercial se mantiene prácticamente constante a lo largo del periodo 1995-2002.

Cuadro 5. Demanda de energía eléctrica en Andalucía según sectores.

\begin{tabular}{|l|l|l|l|l|l|l|}
\hline & \multicolumn{2}{|c|}{ Industrial } & \multicolumn{2}{|c|}{ Comercial } & \multicolumn{2}{c|}{ Residencial } \\
\hline Año & GWh & $\%$ & GWh & $\%$ & GWh & $\%$ \\
\hline 1995 & 6423.48 & $38.49 \%$ & 5255.53 & $31.49 \%$ & 5010.16 & $30.02 \%$ \\
\hline 1996 & 6725.18 & $38.59 \%$ & 5083.78 & $29.17 \%$ & 5618.15 & $32.24 \%$ \\
\hline 1997 & 6963.92 & $38.48 \%$ & 5419.15 & $29.95 \%$ & 5712.66 & $31.57 \%$ \\
\hline 1998 & 7350.44 & $37.99 \%$ & 5870.19 & $30.34 \%$ & 6127.37 & $31.67 \%$ \\
\hline 1999 & 7585.84 & $36.38 \%$ & 6604.12 & $31.67 \%$ & 6663.97 & $31.96 \%$ \\
\hline 2000 & 7931.90 & $35.93 \%$ & 6956.87 & $31.51 \%$ & 7189.59 & $32.56 \%$ \\
\hline 2001 & 8226.07 & $34.97 \%$ & 7466.31 & $31.74 \%$ & 7833.47 & $33.30 \%$ \\
\hline 2002 & 8086.31 & $33.33 \%$ & 7863.32 & $32.41 \%$ & 8309.68 & $34.25 \%$ \\
\hline
\end{tabular}

Fuente. Instituto de Estadistica de Andalucía. Elaboración propia.

La evolución de la estructura de la demanda de electricidad pone de manifiesto el rápido crecimiento que ha experimentado el sector residencial frente al resto de sectores. Esta circunstancia, unida al hecho de que las puntas de demanda de electricidad sean producidas, principalmente, por la intensa variabilidad y el carácter imprevisible que experimenta el consumo doméstico, hace que el mercado residencial sea considerado un sector de especial interés para los planificadores de la demanda de energía eléctrica.

El análisis que nos ocupa pretende evaluar la influencia que tiene la temperatura en la demanda de energía eléctrica, ya que Andalucía, a pesar de tener un clima templado-cálido mediterráneo, se caracteriza por su acusado calor y sequedad estival, e inviernos lluviosos y suaves, siendo generalizada la utilización de aparatos eléctricos para mantener el bienestar de los hogares. La demanda residencial de electricidad en Andalucía fluctúa considerablemente dependiendo de los distintos periodos y condiciones climáticas, destinándose, por término medio, a su consumo uno de cada cuatro kilovatios generados para el mercado doméstico nacional. 
Este trabajo tiene como objeto de estudio la modelización de la demanda residencial mensual de energía eléctrica a partir del efecto que produce la temperatura en el consumo eléctrico.

\section{METODOLOGÍA}

El análisis se desarrolla a partir de los datos históricos en Andalucía durante el periodo 1995-2002. La metodología de estudio esta basada tanto en un enfoque causal como dinámico; orientación, ésta última, hacia la cual se referirá más extensamente este apartado. El análisis se centrará, en primer lugar, en la definición de los principales factores que afectan a la demanda, centrándose la atención en la temperatura. En segundo lugar, se realizará una especificación de los modelos propuestos, detallando las principales características de los mismos, para hacer tras esta etapa la estimación y la evaluación del grado de bondad de ajuste a través de los principales criterios de información.

\subsection{Definición de las variables}

Se han considerado variables representativas de los factores que pudieran afectar a la demanda de energía eléctrica, adquiriendo especial importancia la variable relacionada con la temperatura. A fin de tener en cuenta la influencia de la temperatura como variable exógena, en la ecuación de la demanda de energía se suele considerar una variable denominada en la literatura especializada grados día, que exprese las desviaciones acumulativas de la temperatura respecto a una cierta temperatura neutra durante un periodo determinado. Pueden encontrarse diversas definiciones aunque, en el fondo, todas ellas tienen en común que intentan cuantificar la discrepancia entre la temperatura ambiente, representativa de un período de tiempo, y una cierta temperatura de referencia. En el caso de que esta última coincidiese con la temperatura ambiente, se podría considerar que la temperatura no afecta al consumo residencial de energía eléctrica. En el trabajo no se ha utilizado dicha medida por no estar a disposición pública la temperatura diaria de las provincias andaluzas. En su lugar, y teniendo en cuenta que los datos que, amablemente, nos ha proporcionado el Instituto Nacional de Meteorología se refieren a las temperaturas medias mensuales por provincia, se ha confeccionado una variable representativa de la temperatura media mensual de la Comunidad. 
La determinación cuantitativa de esta "temperatura", calculada a partir de las temperaturas medias de cada provincia, se ha realizado a través de dos procedimientos alternativos que arrojan prácticamente los mismos resultados (véase anexo 1). El primero de ellos pondera la temperatura media de cada provincia según la población de la misma, lo cual es frecuente en la literatura especializada cuando se desea expresar con un único valor la temperatura de una región, a partir de los datos suministrados por estaciones meteorológicas de la misma situadas en provincias de tal región relativamente distantes entre sí.

Por otra parte, y puesto que la ponderación de cada provincia andaluza se puede expresar aproximadamente a través del número de recibos del impuesto de bienes inmuebles, se ha utilizado esta cantidad como factor de ponderación alternativo al correspondiente a la población, $w_{k}$. Este segundo procedimiento es menos redundante con el anterior de lo que pudiera suponerse, pues, aunque los datos corroboran experimentalmente lo que ya a nivel intuitivo cabe esperar a priori, la asociación entre los dos factores de ponderación utilizados no sólo no es proporcional, sino que, en algunas localidades, es de sentido inverso, constituyendo las provincias de Málaga y Sevilla un ejemplo relevante de esto último, pese a que resultan insignificantes las diferencias entre los dos procedimientos en el cálculo de la temperatura media representativa de la Comunidad.

La variable resultante de las consideraciones anteriores se ha denominado grados equivalentes mensuales, Geqmen. Esta variable representa los grados equivalentes mensuales considerando que la temperatura neutra es de $22^{\circ} \mathrm{C}$ y es función de la temperatura media de la Comunidad, $\bar{T}_{i j}$, y del número de días naturales de los cuales consta cada mes, $d_{i j^{\prime}}$

La elección de la temperatura neutra de $22^{\circ} \mathrm{C}$ se justifica mediante un análisis exhaustivo de las distintas combinaciones de modelos según temperaturas en un rango que comprende desde los $15^{\circ} \mathrm{C}$ hasta los $25^{\circ} \mathrm{C}$. El valor de la temperatura neutra considerada, si bien intenta ser plausible, tiene sólo un carácter de instrumento necesario para el estudio metodológico, exento de cualquier interpretación dogmática.

El valor de la variable Geqmen se calcula, para cada mes de los distintos años, como el producto del número de días naturales del mes en cuestión por el valor 
absoluto de la diferencia de la variable anterior definida, temperatura, y la temperatura neutra 22. Su expresión es la siguiente:

$$
\begin{aligned}
& \text { Geqmen }=f\left(T_{i j}, d_{i j}\right)=d_{i j} \times\left|\bar{T}_{i j}-22\right|, \quad i=1,2, \ldots, 12 ; j=1,2, \ldots 8 . \\
& \text { siendo } \bar{T}_{i j}=\stackrel{8}{8}_{k=1}^{8} \bar{T}_{i j k} w_{k} \quad \text { con } \quad \stackrel{\AA}{\mathrm{a}}_{k=1}^{8} w_{k}=1
\end{aligned}
$$

donde $i$ representa los meses del año, $j$ representa los años analizados y $k$ simboliza cada una de las provincias.

La evolución de la demanda de energía eléctrica se comporta según un patrón de tendencia a lo largo del periodo analizado y que se ha recogido, según el modelo, en la variable tiempo, $t$.

La estacionalidad es otra característica de la demanda de electricidad y de la variable Geqmen, no considerándose necesaria la introducción de variables ficticias estacionales. El efecto estacional de la serie ha sido recogido, según el caso, por la variable endógena, por la variable exógena, por la propia tendencia, mediante componentes trigonométricos y los residuos, siendo objeto de análisis en nuestro trabajo.

\subsection{Especificación de los modelos}

La búsqueda de un modelo que exprese la demanda de energía eléctrica a partir de las variables seleccionadas se ha realizado a partir de una regresión lineal uniecuacional, la cual considera modelos de especificación lineal de carácter estático y modelos de especificación lineal de carácter dinámico.

El análisis de los modelos de especificación lineal estática no presenta dificultades, salvo las inherentes al no cumplimiento de las hipótesis del modelo lineal general. Por su parte, la regresión dinámica es un método de predicción que combina los datos que se presentan en forma de serie cronológica con los efectos de variables explicativas 0 indicadores adelantados o precursores. Un modelo de regresión dinámica consta de una variable respuesta, $Y_{t}$, cuyo valor en el tiempo $t$ 
está relacionado linealmente con sus propios valores retardados y con un vector de variables exógenas, $X_{t}$, que está formado por valores correspondientes al período t $y / 0$ precedentes de sus variables componentes $X_{i t}$ suponiéndose habitualmente que las observaciones que han servido para construir la mencionada relación tienen lugar a intervalos igualmente espaciados en el tiempo. Una hipótesis básica en este modelo es que, mientras que la variable endógena está influenciada por las variables independientes, éstas no lo están por la variable endógena, lo que equivale a decir que en este método sólo se consideran modelos uniecuacionales. Una forma general de expresar un modelo regresión dinámica es:

$$
P(B) Y_{t}=\beta X_{t}+\varepsilon_{t}
$$

donde $P(B)$ es un polinomio en el operador de retardo $B, \beta$ es un vector cuyas componentes son los coeficientes de regresión de las $X_{i t}$ (o de sus valores retardados) ${ }_{\mathrm{y}} \mathcal{E}_{t}$ representa el valor del término aleatorio de error en el tiempo $t$; es decir, podría decirse que los modelos de regresión dinámica extienden los modelos ARIMA, por la inclusión de variables exógenas, los modelos ARIMA y los reducen, por la omisión de términos de medias móviles.

El modelo clásico de regresión lineal supone que los errores $\varepsilon_{t}$ correspondientes a dos observaciones cualesquiera de la serie de datos no están correlacionados entre sí. Una solución alternativa para mejorar el modelo dinámico inicial, que generalmente requiere la estimación de menos parámetros que la anterior, la constituye el modelo generalizado de Cochrane-Orcutt, en el que a la ecuación (1) anterior se añade la siguiente:

$$
R(B) \varepsilon_{t}=\varepsilon_{t}-\rho_{1} \varepsilon_{t-1}-\ldots-\rho_{r} \varepsilon_{t-r}=a_{r}
$$

que expresa la autocorrelación de los residuos originales mediante un proceso autorregresivo especificado por el polinomio $R(B)$ en el operador de retardo $B$, siendo $a_{t}$ un proceso de ruido blanco. La ecuaciones (1) y (2) anteriores pueden integrarse en una única ecuación:

$$
R(B)\left[P(B) Y_{t}-\beta X_{t}\right]=a_{t}
$$

Los procedimientos más habituales de estimación de los $\rho_{i}$ en modelos lineales (Cochrane-Orcutt, Prais-Winsten o Hildreth-Lu) son iterativos y suelen estar incorporados en la mayoría de los programas de ordenador. 
Se puede definir una amplia variedad de modelos de regresión dinámica que ayuden a explicar la demanda residencial mensual de energía eléctrica en Andalucía. En este trabajo se presentan aquéllos para los que se obtuvieron mejores resultados desde el punto de vista de su capacidad explicativa.

\subsection{Selección de los modelos}

La elección del modelo más adecuado se puede realizar siguiendo dos enfoques no excluyentes: bien determinando su capacidad explicativa (predicciones dentro de la muestra, exante), bien evaluando su capacidad predictiva (predicciones fuera de la muestra, expost). En el trabajo se utilizan ambos enfoques a través de diversos criterios (véase anexo II) que valoran la precisión de las estimaciones proporcionadas por los modelos candidatos, donde uno de los objetivos en la técnica de selección de modelos es hallar un equilibrio entre la bondad de ajuste y la complejidad de la estructura funcional.

Todos los criterios utilizados se encuentran relacionados entre sí, por depender del Error Cuadrático Medio (ECM), el cual resulta inconsistente como único criterio, ya que no penaliza la inclusión de nuevas variables en el modelo. Sin embargo, a partir del ECM se han utilizado diversos criterios de uso habitual para la selección de modelos, como el Criterio de Información de Akaike (CIA), al cual tiende a sobreparametrizar los modelos elegidos y el Criterio de Información de Schwarz (CIS), que penaliza los modelos más complejos. En este trabajo se utiliza el criterio de Hannan y Quinn (CHQ), como criterio alternativo al CIA, el cual penaliza de forma explícita al tamaño muestral.

Para la evaluación del grado de ajuste de las previsiones, además del error de predicción, se suelen utilizar otros parámetros a los que se les denomina medidas del error de predicción expost, entre las que se han utilizado en este trabajo el Raíz del Error Cuadrático Medio (RECM), Error Absoluto Medio (EAM), Porcentaje de Error Medio Absoluto (PEMA), Coeficiente de Desigualdad de Theil (CDT) y Coeficiente de Janus (CJ). Estos criterios se caracterizan por presentar diversas cualidades. Así, por ejemplo, el RECM y EAM se utilizan para expresar el error en las mismas unidades que la variable objeto de pronóstico. Por otra parte, entre las medidas adimensionales figura el PEMA, dedicada a evaluar la bondad de ajuste en periodos exante, así como la exactitud de la predicción en los periodos extramuestrales. Como medida 
compromiso entre las medidas absolutas y relativas del error de predicción, se emplea el Coeficiente de Desigualdad de Theil (CDT) y el Coeficiente de Janus(CJ), siendo medidas adimensionales, que penalizan los errores elevados de predicción.

\subsection{Combinación de predicciones}

El análisis de la calidad de las predicciones de un modelo no siempre resulta una labor sencilla, ya que, en ocasiones, se puede prescindir de un modelo de predicción por no ser el mejor de acuerdo con el criterio de selección que se haya elegido. Según lo anterior, se puede perder información valiosa contenida en el modelo descartado debido, entre otros motivos, a que algunas variables exógenas contenidas en el modelo descartado pueden no estar incluidas en el supuesto mejor modelo.

La combinación de predicciones, según diversos autores como Clemen, $R$. (1989) y Collopy, F. y Armstrong, J.S. (1992), incrementa la exactitud de los resultados obtenidos de las previsiones individuales de los modelos considerados. Entre las condiciones idóneas de las predicciones a combinar se podrían citar: por un lado, la insesgadez de las previsiones individuales, y por otro, que los errores de predicción individuales estén correlacionados negativamente entre sí, con objeto de que se produjese la mutua cancelación de los mismos.

Un factor importante en la combinación de predicciones es la adecuada elección del coeficiente de ponderación que va a afectar al modelo individual de predicción. En este trabajo se seguirá el procedimiento de asignación de pesos propuesto por Granger (1980) que se expone a continuación.

Supongamos que $f_{n}$ y $g_{n}$ son dos predicciones para el valor $x_{n+1}$, usando dos procedimientos diferentes. En adelante, designaremos a $f_{n}$ y $g_{n}$ simplemente por $f$ y $g$, respectivamente, pues ambas predicciones se supondrán calculadas utilizando los mismos $n$ datos históricos. Una nueva predicción para dicho valor, combinando las predicciones anteriores, sería:

$$
c=k f+(1-k) g \quad 0<k<1
$$


cuyo error de predicción en el periodo $t=n+1$ vendría expresado por:

$e c=x_{n+1}-c=k e f+(1-k) e g$

La varianza de ec viene expresada por:

$$
\operatorname{var}(e c)=k^{2} \operatorname{var}(e f)+(1-k)^{2} \operatorname{var}(e g)+2 k(1-k) \operatorname{cov}(e f, e g)
$$

donde $\operatorname{cov}(e f$, ec es la covarianza de las series ef y eg.

El valor del parámetro $k$ que minimiza la varianza de la serie ec es:

$$
k=\frac{\operatorname{var}(e g)-\operatorname{cov}(e f, e g)}{\operatorname{var}(e g)+\operatorname{var}(e f)-2 \operatorname{cov}(e f, e g)}
$$

Cuando no exista correlación entre ef y eg, esta expresión se reduce a :

$$
k=\frac{\operatorname{var}(e g)}{\operatorname{var}(e g)+\operatorname{var}(e f)}
$$

Si el valor de k obtenido en (5) se sustituye en (4) se demuestra que la varianza del error de la serie combinada nunca será mayor que $\min \{\operatorname{var}(e f)$ var $(e g)\}$.

No obstante, la fórmula (6) no puede ser utilizada para calcular $k$, ya que los valores poblacionales de la varianza y la covarianza que figuran en (5) no son conocidos. Por tanto, estas varianzas se estiman mediante los errores de predicción, empleando este término en el sentido amplio que incluye el periodo de ajuste, tal como se comentó en una sección anterior. De este modo, para calcular el valor de $k$ para el pronóstico combinado del periodo $n+1$ resulta:

$$
k=\frac{\sum_{t=1}^{n}\left(e g_{t}\right)^{2}}{\sum_{t=1}^{n}\left[\left(e g_{t}\right)^{2}+\left(e f_{t}\right)^{2}\right]}
$$

Como se puede observar, el valor de $k$ estará siempre comprendido entre 0 y 1 .

Se puede generalizar esta técnica para combinar $p$ pronósticos $(p \geq 2)$ correspondientes a $p$ métodos diferentes de predicción que proporcionarían el nuevo pronóstico compuesto:

$$
c=k_{1} f_{1}+k_{2} f_{2}+\ldots+k_{p} f_{p}
$$




\section{LA DEMANDA MENSUAL RESIDENCIAL DE ENERGÍA ELÉCTRICA EN ANDALUCÍA Y SU RELACIÓN CON LA TEMPERATURA AMBIENTAL}

Diversos agentes económicos consideran de gran interés la influencia que ejerce la temperatura en el consumo de energía eléctrica, entre los que se podrían citar principalmente: las empresas eléctricas, los analistas económicos y los consumidores. Las empresas eléctricas, porque fuertes variaciones en la temperatura, como la llegada de un frente extremadamente frío u ola de calor, provocan fuertes variaciones en el consumo. Los analistas económicos, porque pueden alterar las tasas de crecimiento, dejando de ser la demanda de electricidad un indicador adecuado para el seguimiento de la actividad económica. En la cadena de suministro eléctrico los consumidores se encuentran en último lugar, sufriendo las consecuencias de una planificación imprecisa de la demanda de energía eléctrica.

En la Gráfico 1 se representa el consumo residencial mensual de electricidad en Andalucía para cada uno de los años de la serie histórica analizada, 1995-2002. En una primera apreciación, se observa que el consumo de energía ha sufrido un crecimiento continuado. Además, los picos de consumo residencial de electricidad tienen lugar durante dos épocas del año bien diferenciadas, una de ellas es la temporada de invierno (diciembre, enero, febrero y marzo) y la otra es la que comprende la temporada de verano (julio, agosto, septiembre). El consumo más elevado de electricidad tiene lugar en el mes de enero para todos los años estudiados desde 1995 hasta 2001, a excepción del año 1996 y 2002 en los que el consumo máximo de electricidad tiene lugar en el mes de marzo y febrero, respectivamente. En enero del año 2002 se registra el nivel máximo de consumo residencial de electricidad con una cantidad de $924,144 \mathrm{GWh}$, con un crecimiento respecto al mismo mes del año anterior del $18,27 \%$.

Por otra parte, puede observarse que el consumo mínimo de electricidad tiene lugar en el mes de junio para la gran mayoría de los años considerados, a excepción de los años 1999, 2000 y 2001, cuyos consumos mínimos se registran en diciembre (años 1999 y 2000) y abril (año 2001), respectivamente. El consumo mínimo de energía eléctrica se produce en junio de 1995, con un volumen de 349,939 GWh. 
Gráfico 1. Consumo mensual de electricidad residencial durante el periodo 1995-2002, expresado en gigavatios por hora(GWh). Fuente. IEA. Elaboración propia.

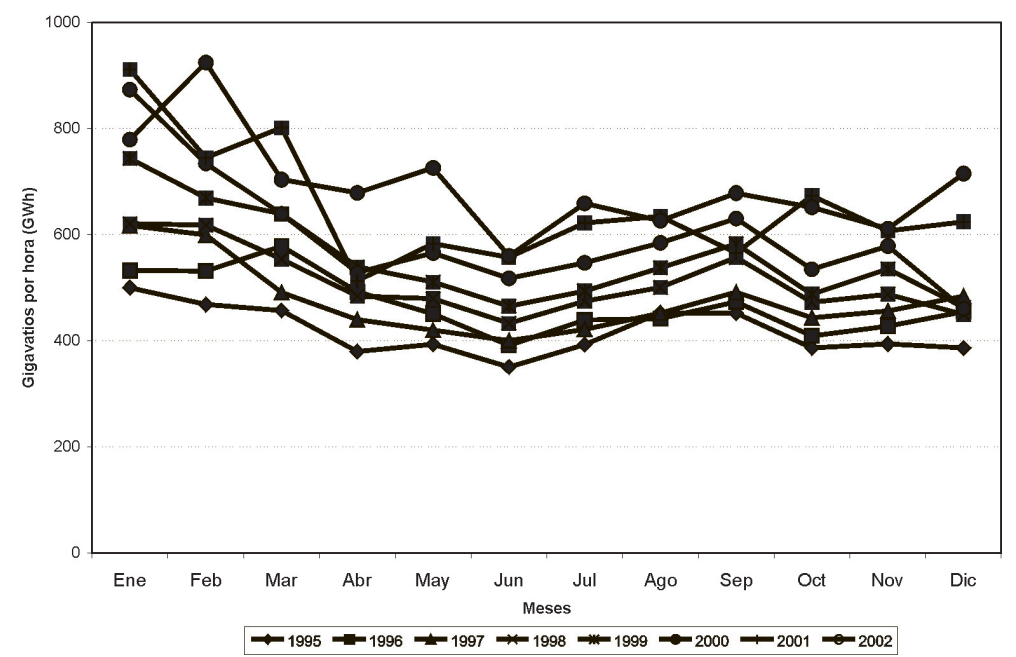

Esta circunstancia de consumos máximos y mínimos en meses con temperaturas no extremas puede ser debida, entre otras causas, a la distinta periodicidad de carácter variable y a la demora con la que se realizan las facturaciones o lecturas de consumo en el periodo correspondiente. Las facturaciones se realizan con periodicidad variable, según sean suministros en alta tensión (mensual) o en baja tensión (bimensual). Por lo tanto, puede suceder que el consumo real de un periodo se corresponda con la facturación producida en el periodo de dos lecturas sucesivas, lo cual es considerado como una situación genérica en las compañías eléctricas. Otro de los factores que pueden afectar a esta circunstancia es la traslación que están sufriendo las estaciones como consecuencia del actual cambio climático.

Si se compara la evolución de la demanda de energía con la evolución de Geqmen, Gráfico 2, se observa un comportamiento muy similar en ambas series. Su conducta describe una morfología de tendencia creciente, motivada, entre otras razones, por el incremento del número de electrodomésticos en los hogares, en su mayor parte, aparatos de calefacción y refrigeración, así como la sustitución del gas butano como energía de uso doméstico. A lo largo del año se observan dos puntas de demanda de distintas intensidades: la primera de ellas y más pronunciada es la que se registra durante la temporada invernal como consecuencia, principalmente, 
de la puesta en marcha de los aparatos de calefacción, y la segunda punta es la que tiene lugar en época estival resultado del funcionamiento intensivo de los aparatos de refrigeración. También se pone de manifiesto la no coincidencia, en todas las ocasiones, de temperaturas máximas o mínimas con consumos máximos, corroborando lo citado en líneas anteriores acerca de la periodicidad variable de las facturaciones.

Sin embargo, si se desagrega el consumo según los registros mensuales, se puede tener mayor grado de detalle de cuál ha sido su evolución en los distintos periodos del año, así como establecer patrones de estacionalidad en el comportamiento de la demanda. Se observa que a partir del año 1998 aumentan las temperaturas extremas, es decir, en el verano se alcanzan temperaturas más altas y en invierno más bajas. El fenómeno anterior repercute en el correspondiente aumento de consumo de electricidad.

Gráfico 2. Consumo mensual de electricidad y grados equivalente $\left(22^{\circ} \mathrm{C}\right)$ durante el periodo 1995 2002. Fuente IEA. Elaboración propia.

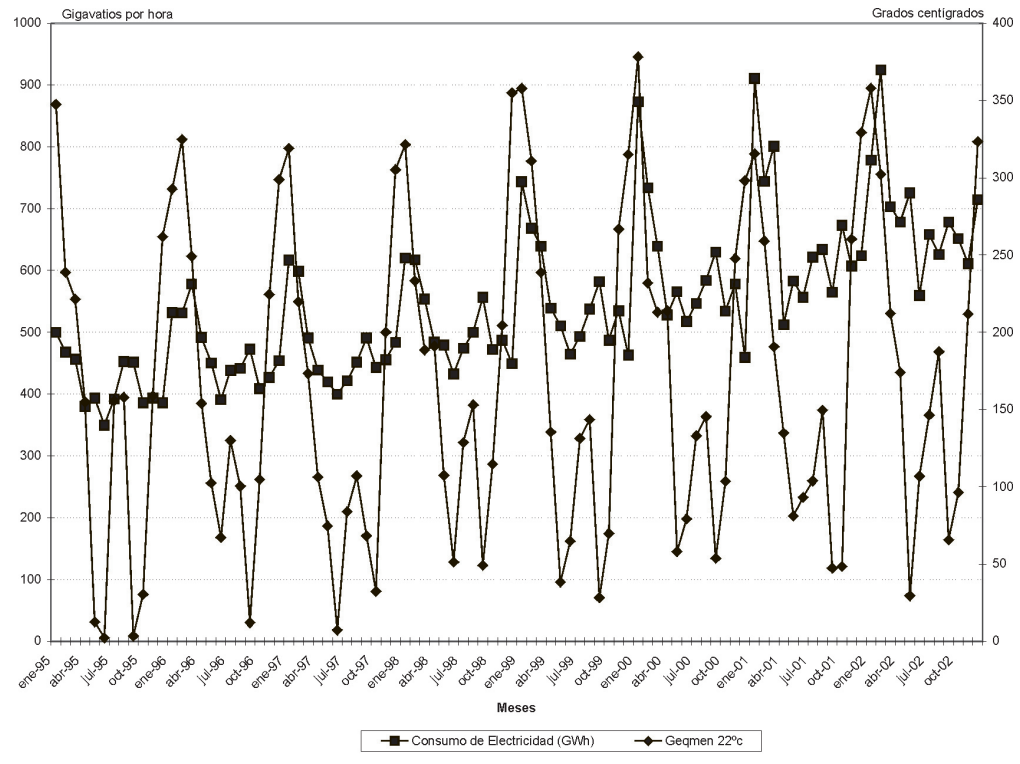

El consumo mensual de electricidad (qmen) se encuentra correlacionado positivamente con respecto a las variables Geqmen y la variable retardada un periodo, Geqmen (-1), representativas de la temperatura. Los coeficientes de correlación para 
el consumo mensual de electricidad y Geqmen son $0,558,0,593^{*} 0,746^{* *}, 0,321$, 0,561, 0,475, 0,546, 0,567 para cada uno de los años del periodo 1995-2002. Si se compara ahora el consumo con Geqmen (-1), se observa cómo en todos los años esta relación es más intensa: $0,675^{\star}, 0,877^{\star *}, 0,851^{\star *}, 0,754^{\star *}, 0,760^{\star *}, 0,615^{\star}, 0,605^{*}$, $0,779^{*}$, lo que confirma empíricamente la existencia de un desfase en la facturación del consumo de electricidad. Además se comprueba que los años 1996 y 2001 son los que registraron un mayor retraso. El nivel de correlación global de la demanda con Geqmen y Geqmen (-1) es de $0,456^{* *}$ y $0,579^{* *}$, respectivamente, siendo correlaciones altamente significativas.

El análisis desarrollado hasta ahora muestra una relación de dependencia de la demanda de electricidad hacia la temperatura. Sin embargo, no necesariamente esta relación tiene carácter de precedencia, en el sentido de causalidad. Para resolver esta cuestión se ha aplicado la prueba de Granger con el objetivo de establecer la naturaleza de la causalidad entre qmen y Geqmen, confirmándose la relación de causalidad en sentido unidireccional, es decir, la temperatura ambiental influye en la demanda de electricidad. La prueba consiste en contrastar, en primer lugar, si Qmensual no causa Geqmen $(0,63)$, así como la hipótesis contraria, es decir, si Geqmen no causa Qmensual (5.6 $10^{-6^{*}}$ ). Los contrastes se aplican sobre uno y dos retardos y los resultados obtenidos coinciden en rechazar la ausencia de relación causal de la temperatura en la demanda residencial de electricidad en la Comunidad Autónoma de Andalucía. Las características generales expuestas de los datos sugieren que la temperatura ambiental debe ser un factor a tener en cuenta a la hora, de estudiar del consumo de electricidad en Andalucía.

\section{MODELIZACIÓN DE LA DEMANDA RESIDENCIAL DE ENERGÍA ELÉCTRICA EN ANDALUCÍA}

La conducta de la demanda de electricidad en Andalucía se encuentra influenciada por la temperatura, resultando ser una variable exógena significativa en todos los modelos que se describen a continuación, seleccionados de entre un número total de 79 modelos analizados.

\footnotetext{
** La correlación es significativa al nivel 0,01.

* La correlación es significativa al 0,05.
} 
En el apartado anterior se ha analizado el perfil de la demanda de electricidad, el cual se caracteriza por una fuerte tendencia creciente, lo que describe una serie no estacionaria según la prueba de Kendall con un estadístico I=0,542 (0,000). Además, se ha observado un patrón estacional, donde los consumos mínimos se registran en los meses de junio y septiembre, siendo la magnitud de las fluctuaciones de la demanda más elevada cuando es creciente, diseñando un patrón heterocedástico en el conjunto de la serie y que se reconoce por un análisis de la variabilidad de las diferencias y cocientes estacionales. Para eliminar el comportamiento heterocedástico de la demanda de electricidad se transforma la serie de demanda original tomando logaritmos, y definiéndose la nueva variable, Qmen. La prueba de raíces unitarias de Dickey-Fuller $(-3,49)$ no confirma la existencia de raíces unitarias de la variable $Q m e n$, al $1 \%$ de nivel de significación.

El proceso de configurar una expresión que ayude a explicar la evolución del consumo eléctrico se inicia con el modelo denominado lineal, que relaciona el consumo de electricidad con la temperatura retardada un periodo y la tendencia, representativa de las variaciones interanuales.

\section{Modelo Lineal: Qmen $_{t}=\beta_{0}+\beta_{1} \operatorname{Geqmen}_{t}(-1)+\beta_{3} t$}

Los resultados del análisis de la regresión muestran la alta significación estadística de la demanda de electricidad; el modelo explica el $82,84 \%$ de la variabilidad del consumo eléctrico. Además sus coeficientes tienen el signo esperado, ya que, manteniendo constante la tendencia, la demanda mensual crece, por término medio, un $0,10 \%$ ante el aumento de un grado centígrado en Geqmen, que traducido a temperatura ambiental se podría interpretar diciendo que un incremento de un grado en la temperatura media mensual supone, por término medio, un crecimiento mensual del $3,15 \%$. La tendencia tiene un efecto positivo, determinante del carácter creciente de la demanda y de un efecto superior al efecto de la temperatura (medido en grados centígrados). La demanda base mensual, sin considerar la temperatura ni la tendencia, es de 343,08 gigavatios por hora y representa aproximadamente la tercera parte de la demanda mensual. La compañías eléctricas deberían asegurar la distribución de este volumen de energía eléctrica en cualquier mes y estación del año El estadístico de Durbin-Watson (DW) no indica un error de especificación en el modelo, ya que su valor es próximo a 2. 
A continuación, se estima un nueva expresión funcional, modelo cuadrático, en el que se ha sustituido la influencia de la temperatura de primer orden por otra de segundo orden. Además no se ha considerado el término de primer grado por no resultar significativo. El modelo cuadrático se define a través de la expresión:

Modelo Cuadrático: Qmen $_{t}=\beta_{0}+\beta_{1} \operatorname{Geqmen}(-1)_{t}^{2}+\beta_{2} t$

El resultado del análisis de la regresión cuadrática es altamente significativo estadísticamente, y la variable exogena, Geqmen y $t$, tienen el signo esperado. El grado de bondad de ajuste es un $2 \%$ superior al modelo lineal, reduciéndose en un 3\% la desviación típica del error. La demanda base ofrecida por el modelo es de 363,22 gigavatios por hora y se reduce considerablemente la influencia de la temperatura en la demanda de electricidad, manteniéndose constante su variación intermensual. Sin embargo, existe riesgo de una autocorrelación negativa de primer orden y, por lo tanto, de una definición errónea del modelo.

Los resultados obtenidos en el modelo cuadrático inducen a pensar que quizás la tendencia por sí sola no recoja ampliamente la variación de la demanda mensual y, por lo tanto, sea necesario complementar el estudio con un análisis armónico, siendo especialmente apropiado para variaciones estacionales. El modelo de regresión sinusoidal considerado para representar las variaciones estacionales presenta un ciclo de seis meses en la demanda de electricidad y se expresa como:

ModeloSinusoidal: Qmen $_{t}=\beta_{0}+\beta_{1} \operatorname{Geqmen}(-1)_{t}+\beta_{2} t+\beta_{3} \operatorname{sen}\left(\frac{4 \pi t}{12}\right)+\beta_{4} \cos \left(\frac{4 \pi t}{12}\right)$

El análisis general de la estimación realizada al modelo sinusoidal, muestra resultados que pueden considerarse satisfactorios. El grado de bondad de ajuste es un 2,08\%, superior al modelo cuadrático y la desviación estándar del error un 3,20\% inferior. Las variables presentan signos correctos en sus coeficientes, la temperatura ejerce un incremento de la demanda mensual del $0,382 \%$, siendo la tasa mensual de crecimiento de la demanda del $0,59 \%$, muy próxima a la obtenida en los modelos anteriores. Este modelo no presenta problemas de autocorrelación de primer orden, por lo que, en términos generales, se podría validar la capacidad explicativa que presenta de la demanda de electricidad.

Hasta ahora, los modelos analizados han seguido un enfoque principalmente causal. Sin embargo, también es importante analizar las posibles relaciones dinámicas que pudieran existir entre la demanda de electricidad actual y la de 
periodos anteriores, así como variables exógenas y perturbaciones. En definitiva, es importante tener en cuenta la información histórica del propio fenómeno objeto de estudio. Para comprobar empíricamente el grado de intensidad de los datos históricos sobre la demanda de electricidad en Andalucía, han sido analizados diversos modelos de especificación dinámica, entre los cuales la estructura más robusta, frente a variaciones de temperatura, es la que se presenta a continuación:

Modelo Dinámico:

Omensual $_{t}=\alpha+\beta_{1}$ qmensual $(-1)+\beta_{2}$ qmensual $(-12)+\beta_{3}$ Geqmen $+\beta_{4} \varepsilon_{t-12}+\beta_{5} \varepsilon_{t-24}$

El modelo es significativo a nivel global y parcial, a pesar de la reducción de los grados de libertad. La estimación del modelo proporciona una mejora sustancial del grado de bondad de ajuste, siendo un 3,5\% superior al modelo sinusoidal. El coeficiente de la variable qmensual (-12) y la temperatura presentan signos correctos, es decir, la demanda de energía eléctrica crece, en términos relativos, con un aumento de la demanda registrada hace un año y cuanto mayor es la diferencia de la temperatura ambiental respecto a la temperatura neutra, siendo ambos efectos independientes. El coeficiente de autocorrelación de los errores presenta valores significativos, mejorando el estadístico DW notablemente respecto al resto de modelos analizados. La única estimación que distorsiona el comportamiento lógico de la demanda es la demanda retrasada un mes. Su coeficiente de regresión tiene signo negativo y de valor muy inferior al de la variable qmensual (-12), lo que produce errores inapreciables en la estimación de la demanda de electricidad. El modelo dinámico incorpora una sustancial mejora desde el punto de vista de la capacidad explicativa de la demanda de electricidad en Andalucía. 
Cuadro 6. Estimación de los modelos lineal, cuadrático, sinusoidal y dinámico.

\begin{tabular}{|c|c|c|c|c|}
\hline Modelos & Lineal & Cuadrático & Sinusoidal & Dinámico \\
\hline$S_{\text {regresion }}$ & 0,1053 & 0,0936 & 0,0906 & 0,0743 \\
\hline Significación & 0,0000 & 0,0000 & 0,0000 & 0,0000 \\
\hline$\beta_{0}$ & 5,8379 & 5,8992 & 5,9147 & 5,9603 \\
\hline$s_{\beta_{0}}$ & 0,0269 & 0,0220 & 0,0213 & 0,0186 \\
\hline Significación & 0,0000 & 0,0000 & 0,0000 & 0,0000 \\
\hline$\beta_{1}$ & 0,0010 & 0,0002 & 0,0038 & 0,0007 \\
\hline$S_{\beta_{1}}$ & 0,0001 & 0,0000 & 0,0001 & 0,0001 \\
\hline Significación & 0,0000 & 0,0000 & 0,0000 & 0,0004 \\
\hline$\beta_{2}$ & 0,0055 & 0,0057 & 0,0511 & 0,0141 \\
\hline$S_{\beta_{2}}$ & 0,0001 & 0,0008 & 0,0140 & 0,0056 \\
\hline Significación & 0,0000 & 0,0000 & 0,0004 & 0,0003 \\
\hline Modelos & Lineal & Cuadrático & Sinusoidal & Dinámico \\
\hline$\beta_{3}$ & - & - & $-0,0448$ & 0,0025 \\
\hline$S_{\beta_{3}}$ & - & - & 0,0134 & 0,0092 \\
\hline Significación & - & - & 0,0012 & 0,0000 \\
\hline$\beta_{4}$ & - & - & & 0,0197 \\
\hline$S_{\beta_{4}}$ & - & - & & 0,0032 \\
\hline Significación & - & - & & 0,0000 \\
\hline$\beta_{5}$ & - & - & & 0,0271 \\
\hline$S_{\beta_{5}}$ & - & - & & 0,0009 \\
\hline Significación & - & - & & 0,0000 \\
\hline $\mathrm{R}^{2}$ ajustado & 0,8284 & 0,8457 & 0,9217 & 0,9542 \\
\hline DW & 2,3513 & 2,6267 & 1,9568 & 2,2329 \\
\hline $\mathrm{CIA}$ & $-1,732$ & $-1,8674$ & $-1,9129$ & $-1,9724$ \\
\hline CIS & $-1,6358$ & $-1,7823$ & $-1,8983$ & $-1,9026$ \\
\hline $\mathrm{ClHQ}$ & $-4,6592$ & $-4,7059$ & $-5,0641$ & $-5,1703$ \\
\hline Estadístico F & 163,6620 & 192,9147 & 175,1507 & 179,6296 \\
\hline
\end{tabular}

Fuente. IEA. Elaboración propia. 
El procedimiento de predicción expost permite reservar los últimos valores observados para evaluar la bondad del ajuste de los modelos, ya que estos valores no se han considerado en el proceso de estimación, siendo las predicciones de estos valores predicciones en sentido estricto. Para realizar la predicción expost se ha contado con la demanda mensual del año 2003. De esta forma, en la cuadro 6 se muestran los valores de los estadísticos que evalúan la capacidad predictiva de cada uno de los modelos para la demanda mensual de energía eléctrica del año 2003. Los modelos lineal y cuadrático son los que peores resultados proporcionan de la predicción de la demanda de electricidad, alcanzando sus errores valores muy distantes de los modelos sinusoidal y dinámico. Por otra parte, el modelo sinusoidal ofrece predicciones más precisas del consumo eléctrico que el modelo dinámico, a diferencia de lo que sucedía en el ajuste del modelo a los datos históricos. La preferencia del modelo sinusoidal se manifiesta en todos los valores de los estadísticos expresados, a excepción del estadístico CJ.

Cuadro 7. Evaluación de la capacidad predictiva de los modelos.

\begin{tabular}{|l|c|c|c|c|}
\hline \multicolumn{1}{|c|}{ Míctele } & Lineal & Cuadrático & Sinusoidal & Dinámico \\
\hline RECM & 0,0627 & 0,0592 & 0,0410 & 0,0437 \\
\hline DMA & 0,0506 & 0,0491 & 0,0382 & 0,0406 \\
\hline PEMA & 0,7569 & 0,7326 & 0,7022 & 0,7190 \\
\hline CDT & 0,0047 & 0,0045 & 0,0040 & 0,0042 \\
\hline CJ & 0,8550 & 0,8430 & 0,8394 & 0,8390 \\
\hline
\end{tabular}

Fuente. IEA. Elaboración propia.

Ante la disyuntiva de despreciar un método de previsión por no ser el mejor de acuerdo con un criterio de selección que se haya elegido, es posible que se esté perdiendo información valiosa contenida en el procedimiento descartado debido, entre otros motivos, a que algunas variables explicativas pueden no estar incluidas en la supuesta mejor predicción. Por todo lo anterior, se propone la combinación de previsiones cuyo fin es incrementar la exactitud de las estimaciones, sin prescindir de la capacidad explicativa del modelo dinámico ni de la capacidad predictiva del modelo sinusoidal proporcionado por ambos. En la cuadro 7 se pueden observar errores de predicciones de los modelos sinusoidal y dinámico, así como la predicción combinada donde se muestra como la predicción combinada reduce el RECM en un $11 \%$, suministrando estimaciones más acertadas. 
Cuadro 8. Errores de predicción de la demanda de energía eléctrica en el año 2003.

\begin{tabular}{|c|c|c|c|}
\hline Mes & Sinusoidal & Dinámico & Predicción Combinada \\
\hline Enero & $-109,225$ & $-1,525$ & $-67,885$ \\
\hline Febrero & 6,026 & $-94,165$ & $-32,432$ \\
\hline Marzo & $-45,453$ & $-129,698$ & $-77,790$ \\
\hline Abril & $-26,470$ & $-24,577$ & $-25,743$ \\
\hline Mayo & $-31,921$ & 4,938 & $-17,773$ \\
\hline Junio & $-39,981$ & $-16,817$ & $-31,090$ \\
\hline Julio & $-5,556$ & $-4,827$ & $-5,276$ \\
\hline Agosto & 7,047 & $-59,665$ & $-18,560$ \\
\hline Septiembre & $-37,466$ & $-35,927$ & $-36,875$ \\
\hline Octubre & $-41,397$ & 13,031 & $-20,505$ \\
\hline Noviembre & 4,194 & 13,942 & 7,936 \\
\hline Diciembre & $-15,902$ & $-39,731$ & $-25,049$ \\
\hline RECM & 41,652 & 52,772 & 37,081 \\
\hline
\end{tabular}

Fuente. IEA. Elaboración propia.

En la Gráfico 3 se muestra una comparación gráfica de las previsiones de la demanda de electricidad que realizan los métodos sinusoidal, dinámico y combinado en el año 2003. La estimación confidencial del modelo combinado expresa un ajuste adecuado del modelo, quedando la demanda real de electricidad dentro del intervalo en todos los meses. 
Gráfico 3. Comparación de las previsiones de los modelos sinusoidal, dinámico y combinado con la demanda real de electricidad. Fuente. IEA. Elaboración propia.

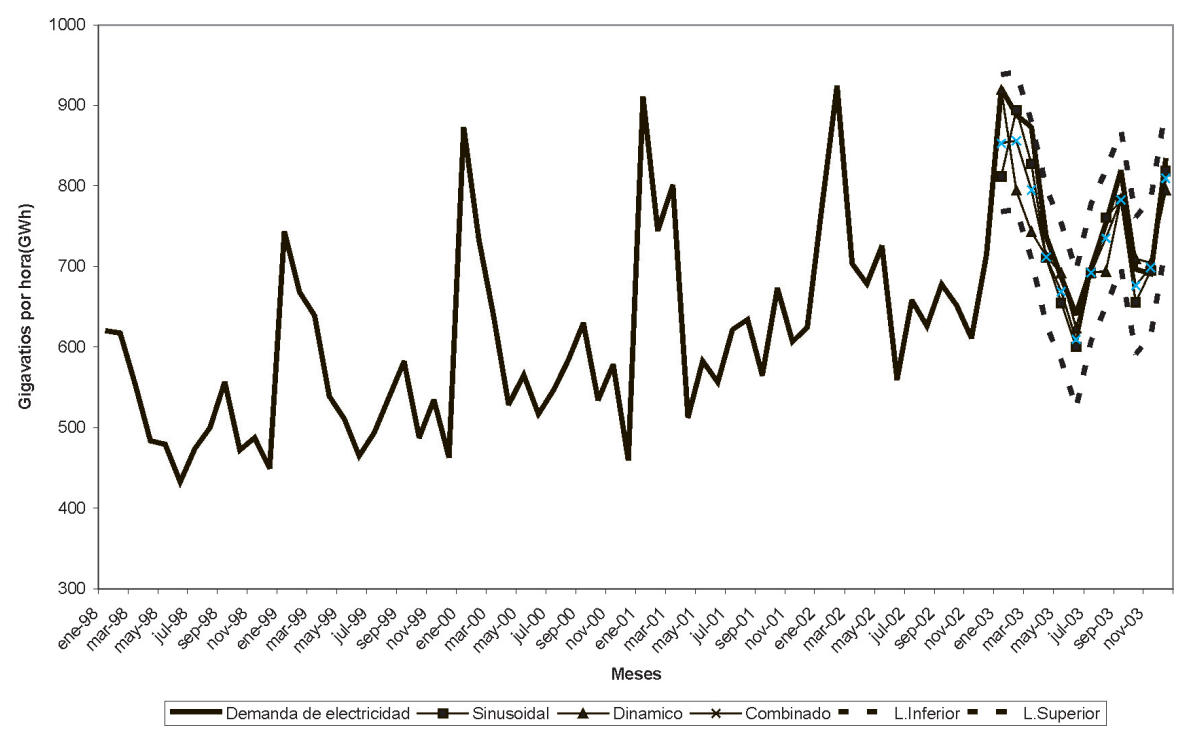

\section{CONCLUSIONES}

La calidad del suministro eléctrico es uno de los requisitos fundamentales que ha de cumplir todo sistema eléctrico, y, como tal, los planificadores deben asegurar su continuidad en los distintos escenarios temporales. Todo lo anterior determina la necesidad de emplear mecanismos que reduzcan el riesgo de ocasionar consecuencias no deseables a sus usuarios, en especial, al mercado doméstico por contar con principios más imprevisibles. Siguiendo esta línea, en el presente trabajo se propone un modelo de previsión de la demanda mensual de energía eléctrica en Andalucía, el cual pudiera ser extensible al resto de regiones españolas.

Este análisis se ha realizado considerando la influencia que ejerce la temperatura en la variación del consumo eléctrico, requiriéndose la construcción de un indicador global de la temperatura. Este indicador ha sido elaborado a partir del estudio exhaustivo del comportamiento del consumo de electricidad en las distintas épocas del año, llegándose a la conclusión de que los niveles mínimos de demanda se alcanzan cuando la temperatura se encuentra próxima a los $22^{\circ} \mathrm{C}$ y que cualquier variación respecto a esta temperatura intensifica el uso de los aparatos de calefacción 
0 de refrigeración. A partir de las temperaturas medias se ha construido la variable Geqmen, que representará en el modelo de previsión las desviaciones mensuales de temperatura ambiental respecto a la temperatura neutra. Asimismo, la metodología utilizada ha permitido aclarar ciertos aspectos relacionados con el sistema de ponderación elegido para la definición de Geqmen.

En este sentido, considerando la representatividad del estudio realizado y su limitación geográfica, se podrían sintetizar los resultados de esta investigación a través de las siguientes conclusiones:

- La evolución de la demanda de electricidad durante el periodo 1995-2002 ha tenido un carácter marcadamente creciente. Hay que destacar que la tasa de variación interanual es creciente, especialmente a partir del año 1999.

- Se ha utilizado como factor de ponderación de la temperatura mensual, además del número habitantes, el número de inmuebles con relación de propiedad perfectamente delimitada a efectos fiscales, proporcionando valores similares de la temperatura media mensual de la Comunidad.

- En el análisis conjunto de la demanda de electricidad y la temperatura se observa una trayectoria caracterizada por la intensa estacionalidad de las observaciones, así como por la fuerte correlación existente entre el consumo y la temperatura durante los ocho años analizados. Los consumos máximos tienen lugar en dos épocas del año bien diferenciadas: una de ellas coincide con la época invernal y la otra con la época estival, registrando esta última niveles de consumo inferiores a los registrados en la temporada de invierno. Además, se ha observado que, especialmente en los últimos años, el consumo máximo no siempre se corresponde con una temperatura máxima o mínima, lo que nos ha llevado a interpretar que este comportamiento es consecuencia de la demora en la facturación que presentan la mayoría de las compañías eléctricas. Este hecho ha sido de especial importancia en la especificación de un modelo de previsión de la demanda de electricidad.

- A partir de los modelos de regresión analizados cuya especificación es de carácter lineal, cuadrática, sinusoidal y dinámica, se ha demostrado analíticamente la significatividad de la temperatura en la demanda de electricidad. Según el modelo, esta influencia es inferior a la propia tendencia de la serie y al comportamiento estacional de la demanda. 
- Se ha evidenciado analíticamente que el modelo con mejor capacidad explicativa es el modelo de regresión dinámica con un nivel de ajuste del $95,42 \%$, apoyando esta afirmación todos los estadísticos considerados. De esta forma, la demanda de energía eléctrica de un mes depende, junto con la temperatura, del consumo del mes anterior y del consumo que para ese mismo mes se registró en el año anterior.

- Según la mayoría de los criterios, el modelo sinusoidal es el que proporciona estimaciones expost más precisas, a pesar de que estas estimaciones, según el criterio $\mathrm{CJ}$, sean menos exactas en el modelo sinusoidal que en el modelo dinámico.

- La combinación de las previsiones del modelo dinámico y sinusoidal proporciona un modelo de previsión de calidad, ya que el error de predicción para el año 2003 se reduce en un $11 \%$ respecto a las previsiones realizadas con el modelo sinusoidal.

\section{BIBLIOGRAFÍA}

Adams,G.; Allen,P.G. y Morzuch, J. (1991): "Probability Distributions of Short-Term Electricity Peak Load Forecasts", International Journal of Forecasting, 7, 3, págs. 283-298.

Ayyash, S.; Salman, M. y Al-Hafi, N. (1985): "Modelling the impact of temperature on summer electricity consumption in Kuwait", Energy-The International Joournal, 10, 8, págs.941-49.

Boletines Estadísticos Mensuales de Energía Eléctrica. Red Eléctrica Española.

Bolzern, P.; Fronza, G. y Brusasca, G. (1982): "Temperature effects on the winter daily electric load”, Journal of Applied Meteorology, 21, 2.

Box, G.E.P. y Jenkins. G.M. (1976): "Time Series Analysis: Forecasting and Control", Holden Day .

Bunn, D.W. y Farmer, E.D. (1985): "Comparative Models for Electrical Load Forecasting", Wiley. 
Cancelo, J.R. y Espasa. A. (1991): "Un nuevo indicador semanal y mensual de actividad basado en el consumo de energía". Documento de Trabajo 91-06. Departamento. Economía, Universidad Carlos III de Madrid.

Cancelo, J.R.; Cancelo, J.R. y Espasa. A. (1995): "Modelización del efecto temperatura en el consumo de electricidad". Estadística Española, vol. 37, num. 139, págs. 183200.

Clemen, R. (1989): "Combinig Forecast: a review and annotated bibliography", International Journal of Forecasting, 5, págs.559-584.

Collopy, F. y Armstrong, J.S. (1992): "Expert Opinions about Extrapolation and the Mystery of the Overlooked Discontinuities", International Journal of Forecasting, 8, 4, págs.575-582.

Davies, M., (1959): "The Relationship between Weather and Electricity Demand", Proc.IEEE, 106C, págs.27-37.

Dryar, H.A. (1944): "The effect of weather on the system load". Trans AIEE, 63, págs.1006-1013.

Engle, R., Ramanathan, R. Granger, C.W.J, Vahid-Araghi, F. y Brace, C. (1997): "Short-run forecast of electricity loads and peaks. International Journal of Forecasting, 13, págs.161-174.

Faruqui, A. (1987): "On the Search for Accuracy in Electric Utility Forecasting", Journal of Forecasting, April , págs.93-96.

Fischler, E.B. y Nelson, R.F. (1986): "Integrating Time Series and End Use Methods of Forecasting Electricity Sales", Journal of Forecasting, January, págs.15-30.

Gallástegui, I. (1986): "Análisis de Series Temporales: Algunas Técnicas de Predicción", EUSTAT.

Granger, C.W.J. (1980): "Forecasting in Business and Economics". Academic Press.

Gutiérrez Moya, E. (2003): "La demanda residencial de energía eléctrica en Andalucía: un análisis cuantitativo". Tesis Doctoral. Servicio de Publicaciones de la Universidad de Sevilla. 
Harvey, A.C. y Koopman, S.J. (1993): "Forecasting hourly electricity demand using time-varing splines. Journal of American Statistical Association, 88, págs.1228-1236.

Hipper, H.S.; Pedreira, C.E. y Souza, R.C. (2001): "Neural networks for short term load forecasting a review and evaluation". IEE Transactions on Power Systems, 16, págs. 44-45.

Le Comte, D.M. y Warren, H.E. (1981): "Modelling the Impact of Summer Temperatures on National Electricity Consumption", Center for Environmental Assessment Services, Washington.

Mabert, V. A. y Radcliffe, R. C. (1974): "A Systematic Modeling Methodology". Financial Management, Autumn, págs.59-67.

Makridakis, S. (1984): "The Forecasting Accuracy of Major Time Series Methods", Wiley.

Nelson,C.R. (1984): "A Benchmark for the Accuracy of Econometric Forecasts of GNP", Business Economics, 19,3, págs. 52-58.

Pankratz, A. (1991): "Forecasting with Dynamic Regression Models", Wiley Interscience Publication.

Quayle, R.G. y Diaz, H.F. (1980): "Heating degrees day data applied to residential heating energy consumption" J. Applied Meteor,19, págs. 241-248.

Sanz, R. (1979): "Modelización del indice de producción industrial y su relación con el consumo de energía", Cuadernos Económicos de ICE, 11-12, págs. 249-259.

Stanton, K.N. y Gupta, P.C. (1969): "Forecasting annual or seasonal peak demand in electric utility systems". IEEE paper 69 TP 648.PWR, Summer Power Meeting, 22-27 junio.

Taylor, L.D. (1975): "The Demand for Electricity: A Survey", Bell Journal of Economics, Spring, págs. 74-110.

Taylor, J.W. y Buizza, R. (2003): "Using weather ensemble predictions in electricity demand forecasting". International Journal of Forecasting, 19, págs. 57-70. 
Thompson, R.P. (1976):"Weather Sensitive Electric Demand and Energy Analysis on a Large Geographically Diverse Power System - Application to short term hourly electric demand forecasting", IEEE Transactions on Power Apparatus and Systems, Vol. PAS95, no. 1, January/February.

Temperaturas Mínimas, Máximas y Medias Mensuales por provincia de la Comunidad Autónoma de Andalucía, años 1995-2001. Centro Meteorológico Territorial en Andalucía Occidental y Ceuta.

Williams y Leslie. (1953): "Load forecasts reflect weather", Electr. World,139, págs. 86-88. 
ANEXO I

Temperatura ponderada según población, impuesto de bienes inmuebles y promedio

\begin{tabular}{|c|c|c|c|c|c|c|c|}
\hline mes-año & según población & según IBI & promedio población e IBI & mes-año & según población & según IBI & promedio población e IBI \\
\hline ene-95 & 10,792 & 10,776 & 10,704 & ene-99 & 10,457 & 15,672 & 15,688 \\
\hline feb-95 & 13,472 & 13,414 & 13,442 & feb-99 & 10,900 & 16,752 & 16,773 \\
\hline mar-95 & 14,856 & 14,747 & 14,800 & mar-99 & 14,297 & 19,557 & 19,616 \\
\hline abr-95 & 16,835 & 16,682 & 16,661 & abr-99 & 17,484 & 23,442 & 23,482 \\
\hline may-95 & 21,593 & 21,480 & 21,533 & may-99 & 20,765 & 26,448 & 24,841 \\
\hline jun-95 & 22,076 & 23,069 & 23,094 & jun-99 & 24,160 & 30,316 & 30,341 \\
\hline jul-95 & 27,021 & 26,944 & 26,979 & jul-99 & 26,241 & 32,531 & 32,523 \\
\hline ago-95 & 27,096 & 27,043 & 27,066 & ago-99 & 26,629 & 32,775 & 32,776 \\
\hline sep-95 & 22,116 & 21,741 & 21,791 & sep-99 & 22,946 & 28,235 & 28,227 \\
\hline oct-95 & 21,018 & 20,877 & 20,946 & oct-99 & 19,747 & 23,775 & 23,784 \\
\hline nov-95 & 16,702 & 16,529 & 16,636 & nov-99 & 13,108 & 18,085 & 18,121 \\
\hline dic-95 & 13,554 & 13,462 & 13,508 & dic-99 & 11,835 & 15,900 & 15,926 \\
\hline ene-96 & 12,556 & 12,475 & 12,516 & ene- 00 & 9,799 & 14,959 & 14,985 \\
\hline feb-96 & 10,797 & 10,724 & 10,689 & feb-00 & 14,004 & 20,379 & 20,409 \\
\hline mar-96 & 13,963 & 13,837 & 13,900 & mar-00 & 15,129 & 21,001 & 21,063 \\
\hline abr-96 & 16,865 & 16,719 & 16,791 & abr-00 & 14,870 & 19,417 & 19,430 \\
\hline may-96 & 18,692 & 18,541 & 18,615 & may- 00 & 20,125 & 25,287 & 25,314 \\
\hline jun-96 & 24,241 & 24,082 & 24,158 & jun-00 & 24,640 & 30,652 & 30,689 \\
\hline jul-96 & 26,194 & 26,088 & 26,138 & jul-00 & 26,288 & 32,711 & 32,728 \\
\hline ago-96 & 25,239 & 25,183 & 25,209 & ago-00 & 26,691 & 32,988 & 33,015 \\
\hline sep-96 & 21,596 & 21,513 & 21,554 & sep-00 & 23,790 & 29,631 & 29,674 \\
\hline oct-96 & 18,620 & 18,488 & 18,553 & oct- 00 & 18,654 & 23,653 & 23,708 \\
\hline nov-96 & 14,518 & 14,400 & 14,459 & nov- 00 & 13,741 & 18,035 & 18,056 \\
\hline dic-96 & 12,359 & 12,245 & 12,303 & dic-00 & 12,384 & 18,171 & 18,353 \\
\hline ene-97 & 11,709 & 11,593 & 11,652 & ene-01 & 11,818 & 11,732 & 11,776 \\
\hline feb-97 & 14,150 & 14,015 & 14,083 & feb-01 & 12,746 & 12,621 & 12,684 \\
\hline mar-97 & 16,412 & 16,203 & 16,306 & mar-01 & 15,847 & 15,774 & 15,809 \\
\hline abr-97 & 18,456 & 18,279 & 18,366 & abr-01 & 17,507 & 17,395 & 17,449 \\
\hline may-97 & 19,591 & 19,509 & 19,548 & may-01 & 19,382 & 19,278 & 19,328 \\
\hline jun-97 & 22,243 & 22,232 & 22,235 & jun-01 & 25,110 & 25,051 & 25,077 \\
\hline jul-97 & 24,709 & 24,635 & 24,363 & jul-01 & 25,351 & 25,365 & 25,355 \\
\hline ago-97 & 25,455 & 25,420 & 25,809 & ago-01 & 26,824 & 26,822 & 26,819 \\
\hline sep-97 & 24,274 & 24,136 & 24,204 & sep-01 & 23,580 & 23,543 & 23,559 \\
\hline oct-97 & 20,955 & 20,845 & 20,941 & oct-01 & 20,435 & 20,354 & 20,394 \\
\hline nov-97 & 15,330 & 15,215 & 15,273 & nov-01 & 13,323 & 13,239 & 13,281 \\
\hline dic-97 & 12,156 & 12,086 & 12,122 & dic-01 & 11,381 & 11,278 & 11,341 \\
\hline ene-98 & 11,631 & 11,559 & 11,596 & ene-02 & 12,023 & 11,913 & 11,968 \\
\hline feb-98 & 13,669 & 13,510 & 13,589 & feb-02 & 12,963 & 12,884 & 12,924 \\
\hline mar-98 & 15,914 & 15,730 & 15,821 & mar-02 & 15,028 & 14,885 & 14,956 \\
\hline abr-98 & 15,632 & 15,573 & 15,621 & abr-02 & 16,322 & 16,193 & 16,258 \\
\hline may-98 & 18,532 & 18,439 & 18,484 & may-02 & 19,293 & 19,204 & 19,249 \\
\hline jun-98 & 23,711 & 23,621 & 23,664 & jun-02 & 23,985 & 23,989 & 23,987 \\
\hline jul-98 & 26,153 & 26,564 & 25,926 & jul-02 & 26,018 & 25,980 & 25,999 \\
\hline ago-98 & 26,935 & 26,855 & 26,893 & ago-02 & 25,062 & 25,057 & 25,059 \\
\hline sep-98 & 23,640 & 23,587 & 23,612 & sep-02 & 22,442 & 22,416 & 22,429 \\
\hline oct-98 & 18,301 & 18,158 & 18,229 & oct- 02 & 19,543 & 19,437 & 19,490 \\
\hline nov-98 & 15,186 & 15,091 & 15,139 & nov-02 & 15,116 & 15,062 & 15,089 \\
\hline dic-98 & 10,554 & 10,457 & 10,507 & dic-02 & 12,745 & 12,766 & 12,756 \\
\hline
\end{tabular}




\section{ANEXO II}

Estadísticos evaluadores de la capacidad explicativa:

- Coeficiente de Determinación: $\bar{R}^{2}=1-\frac{s^{2}}{\sum_{t=1}^{n}\left(y_{t}-\bar{y}_{t}\right)^{2} / n-1}$

- Criterio de Información de Akaike: $C I A=\ln \left(\frac{\sum_{t=1}^{n} e_{t}^{2}}{n}\right)+\frac{2 k}{n}$

- Criterio de Información de Schwarz: $C I S=\ln \left(\frac{\sum_{t=1}^{n} e_{t}^{2}}{n}\right)+\frac{k \ln (n)}{n}$

- Criterio de Información de Hannan y Quinn:

$$
C I H Q=\ln \left(\frac{\sum_{t=1}^{n} e_{t}^{2}}{n}\right)+\frac{2 \ln [\ln (n) k]}{n}
$$

\section{Estadísticos evaluadores de la capacidad predictiva:}

- Raíz del Error Cuadrático Medio: RECM $=\sqrt{\frac{1}{m} \sum_{t=n+1}^{n+m}\left(y_{t}-\hat{y}_{t}\right)^{2}}$

- Desviación Media Absoluta: $D M A=\frac{1}{m}\left\{\sum_{i=n+1}^{n+m}\left|y_{t}-\hat{y}_{t}\right|\right\}$

- Porcentaje de Error Medio Absoluto: $D M A=\frac{1}{m}\left\{\sum_{t=n+1}^{n+m}\left|y_{t}-\hat{y}_{t}\right|\right\}$ 
- Coeficiente de Desigualdad de Theil: PEMA $=\frac{1}{m} \sum_{t=n+1}^{n+m}\left|\left(\frac{y_{t}-\hat{y}_{t}}{y_{t}}\right) \cdot 100\right|$

- Coeficiente de Janus: $C J=\sqrt{\frac{\sum_{t=n+1}^{n+m}\left(\hat{y}_{t}-y_{t}\right)^{2}}{m}}$ 